\title{
Prox1 dosage controls the number of lymphatic endothelial cell progenitors and the formation of the lymphovenous valves
}

\author{
R. Sathish Srinivasan and Guillermo Oliver ${ }^{1}$ \\ Department of Genetics, St. Jude Children's Research Hospital, Memphis, Tennessee 38105, USA
}

\begin{abstract}
Arteries, veins, and lymphatic vessels are functionally linked, and their physical interaction is tightly regulated. The lymphatic vessels communicate with the blood vessels only at the junction of the jugular and subclavian veins. Here, we characterize the embryonic lymphovenous valves controlling this vital communication and show that they are formed by the intercalation of lymphatic endothelial cells (LECs) with a subpopulation of venous endothelial cells (ECs) at the junction of the jugular and subclavian veins. We found that unlike LEC progenitors, which move out from the veins and differentiate into mature LECs, these Prox1-expressing ECs remain in the veins and do not acquire LEC features. We demonstrate that the development of this Prox1-expressing venous EC population, and therefore of lymphovenous valves, requires two functional copies of Prox1, as the valves are absent in Prox1 heterozygous mice. We show that this is due to a defect in the maintenance of Prox1 expression in venous ECs and LEC progenitors promoted by a reduction in Coup-TFII/Prox1 complex formation. This is the first report describing the molecular mechanism controlling lymphovenous communication.
\end{abstract}

[Keywords: LEC progenitors; Prox1; lymphatics; lymphovenous valves]

Supplemental material is available for this article.

Received May 6, 2011; revised version accepted September 6, 2011.

In mammals, the two vascular systems-the blood vasculature, which transports oxygen and nutrients, and the lymphatic vasculature, which transports fluid and macromolecules from tissues back to the blood circulation-are tightly interconnected. Because of differences in oncotic pressure, the plasma fluids that continuously extravasate from the blood vasculature are collected from the tissue space by the lymphatic vasculature that then returns it back to the blood circulation so that fluid homeostasis is properly maintained (Oliver and Srinivasan 2008).

The stepwise process leading to the formation of the lymphatic vascular network starts when differentiating Prox1-expressing lymphatic endothelial cell (LEC) progenitors leave the embryo's cardinal vein $(\mathrm{CV})$ to form intermediate structures called lymph sacs. The first lymph sacs to develop are the paired jugular lymph sacs. Each jugular lymph sac retains a connection to the adjacent vein (Sabin 1902; Kampmeier 1969; van der Putte 1975) controlled by lymphovenous valves (van der Putte 1975). Because this connection is where reabsorbed tissue fluids collected by the lymphatics are released back into the blood circulation, any alteration in the genesis of the lympho-

${ }^{1}$ Corresponding author.

E-mail guillermo.oliver@stjude.org.

Article is online at http://www.genesdev.org/cgi/doi/10.1101/gad.16974811. venous valves is supposed to severely compromise the maintenance of normal fluid homeostasis.

The anatomical identification of these lymphovenous valves has been hampered by the lack of specific makers (Lewis 1905; Gittenberger-De Groot et al. 2004) and no information is yet available about the molecular and cellular mechanisms leading to their formation. However, in the last decade, genes controlling different aspects of developmental and postnatal lymphangiogenesis have been identified, and useful animal models have been generated (Oliver and Srinivasan 2008). Taking advantage of these tools, we have now performed a detailed molecular characterization of these lymphovenous valves during developmental lymphangiogenesis. We conclusively show that the lymphovenous connection is mediated by two nearby valves located at the junction of the jugular and subclavian veins. We report for the first time that these valves are formed by the intercalation of lymph sac-derived Prox $1^{+}$LECs with a previously unidentified population of Prox $1^{+}$endothelial cells (ECs) present in the adjacent veins. As LECs originate from the embryonic veins (Srinivasan et al. 2007), we argue that these Prox $1^{+}$ECs arise from a subpopulation of the Proxl-expressing venous ECs; some will become LEC progenitors and leave the vein, while others will remain on the vein and participate in the formation of the lymphovenous valves. We also show that in Prox1 heterozygous embryos, the number of Prox1- 


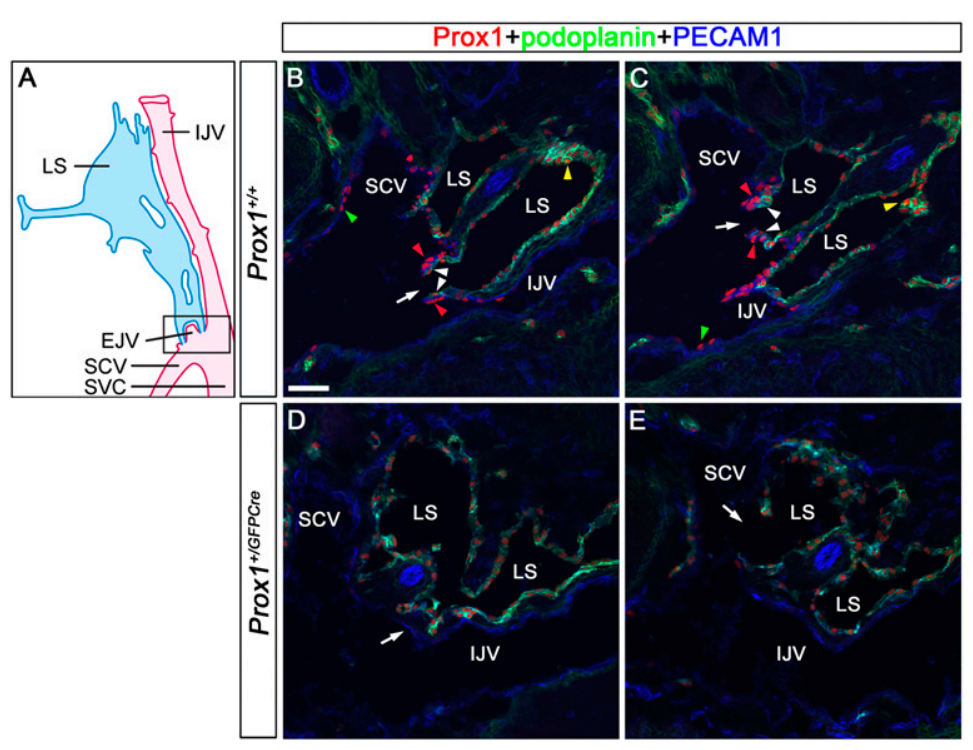

Figure 1. Prox1 is expressed on a previously unidentified population of ECs in the lymphovenous valves. $(A)$ Schematic representation (adapted with modifications from van der Putte, 1975) of the area of an E13.5 embryo where the jugular and subclavian veins join to form the lymphovenous valves. The head (anterior) is oriented toward the top and the heart (posterior) is oriented toward the bottom of the figure. These veins merge to form the SVC. The posterior portion of the jugular lymph sac (LS) opens into the SCV where the veins merge. To visualize the boxed region of the diagram, E13.5 wildtype $(B, C)$ or Prox $1^{+/ G F P C r e}(D, E)$ embryos were frontally sectioned from the dorsal to the ventral side and immunostained for the LEC markers Proxl and podoplanin and the pan-endothelial PECAM1. (B) The first valve (arrow) is adjacent to the IJV on one side and the SCV on the other side. $(C)$ The second valve (arrow) in this orientation is on the SCV. Each of the valve's two leaflets consists of two layers of Prox $1^{+}$ECs: an inner Prox $1^{+}$ podoplanin ${ }^{+}$layer that is continuous with the lymph sac (white arrowheads) and an outer Prox $1^{+}$podoplanin ${ }^{-}$ layer that is continuous with the veins (red arrowheads). Note the relatively high levels of Proxl in the valves (red arrowheads) and in some cells of the lymph sac (yellow arrowheads). (B,C) Few Prox $1^{+}$cells are also seen on the veins away from the lymphovenous valves (green arrowheads). (D) In Prox $1^{+/ G F P C r e}$ embryos, Prox 1 is not expressed on the walls of the veins (arrow). (E) Occasionally, the lymph sacs abruptly fuse with the IJV (arrow). The brain is oriented toward the right, the heart is oriented toward the left, and the thymus is oriented toward the bottom of $B-E$. Bar, $50 \mu \mathrm{m}$.

expressing ECs on the vein is reduced, and, consequently, the number of LECs is reduced and the lymphovenous valves fail to form. We demonstrate that this defect is because of a reduction in the amount of Coup-TFII/Prox1 complex formation necessary for the maintenance of Prox 1 expression. We thereby provide a mechanistic explanation for the haploinsufficiency observed in Prox 1 heterozygous mice (Harvey et al. 2005).

\section{Results}

\section{Molecular identification and characterization of the lymphovenous valves}

Previous detailed anatomical studies in mouse embryos identified the junction of the jugular and subclavian veins as the location where the communication between the lymphatic and blood vasculatures is established (Fig. 1A; van der Putte 1975). Internal and external jugular veins return the deoxygenated blood from the brain and other anterior organs to the heart, and the subclavian vein (SCV) returns deoxygenated blood from the limb buds (van der Putte 1975). The internal jugular vein (IJV) runs in an anterior to posterior orientation, and the SCV runs more or less perpendicular to the IJV (Fig. 1A). The external jugular vein (EJV) takes a lateral route after leaving the skull, and because of its orientation only the site of its fusion with the $\mathrm{SCV}$ is represented in the scheme shown in Figure 1A. These three veins merge at approximately the level of the thymus to form the superior vena cava (SVC), which in turn opens into the atrium of the heart (van der Putte 1975). All three veins are symmetrically paired structures with one vein of each pair present on each side of the body (van der Putte 1975).
Taking this anatomical information into account, we performed a detailed anatomical and molecular characterization of the formation of the lymphovenous valves in mouse embryos. First, embryonic day 13.5 (E13.5) embryos were frontally sectioned (dorsal to ventral) to facilitate the visualization of the lymph sacs along with the IJV, the SCV, and the valves through which they interact (Fig. 1A). Then, sections were coimmunostained using the LEC markers Prox 1 and podoplanin and the pan-endothelial marker PECAM1. As expected, two valves connecting the lymph sacs with the surrounding veins were identified at the junction between the jugular and subclavian veins (Fig. $1 \mathrm{~B}, \mathrm{C}$, arrows). One of these valves lies more dorsomedially than does the other one (Fig. 1B, arrow). Veins and lymph sacs are nearly symmetrically located on the left and right sides of the embryo. Similarly, the lymphovenous valves are also present on both sides, and their locations and overall structures are nearly identical (data not shown).

We found that each valve is composed of two leaflets, each containing two layers of Prox $1^{+}$cells. The inner layer is contributed by the lymph sacs; therefore, it is composed of Prox $1^{+}$podoplanin ${ }^{+}$LECs (Fig. 1B,C, white arrowheads). However, the outer layer is composed of a previously unidentified population of PECAM $1^{+} / \operatorname{Prox~}^{+} /$podoplanin $^{-}$ECs extending from the walls of the surrounding veins (Fig. 1B,C, red arrowheads).

To better understand the structural organization of these valves, we performed immunostainings for Prox 1 and PECAM1 on transverse sections along the anterior-posterior axis. As expected, Prox 1 expression is detected lining the entire lymph sac at the most anterior level but is not observed on the IJV (Supplemental Fig. S1A). However, as we move posterior to the region shown within the box in Figure 1A, Proxl expression is detected on the IJV's wall 
but only within the region that is in closest contact with the lymph sac (Fig. 2A, arrow). Proxl is detected on the walls of the IJV and the SCV as the SCV approaches the jugular vein (Supplemental Fig. S1B). Around this level, where the lymph sac is split into two portions by the vertebral artery (Fig. 2B, arrowhead), the more medial segment intercalates with Prox $1^{+}$cells originating from the IJV on one side and from the SCV's wall on the other side (Fig. 2B, arrows). This structure corresponds to the first valve shown on the frontal sections of Figure 1A. Posterior sections show that the IJV and the SCV are fused together, forming a single blood vessel containing a cluster of Prox $1^{+}$ cells representing the tip of the valve (Fig. 2C, arrow). The more lateral portion of the lymph sac lies close to the SCV (Fig. 2D). The EJV, which was not apparent in the frontal sections, becomes obvious in adjacent sections where it branches off from the SCV and moves laterally (Fig. 2E, arrow). High levels of Proxl expression are detected on the EC located on the wall of the EJV that is in close contact with the wall of the Proxl-expressing lymph sac (Fig. 2E). Because of the close proximity between these two structures (i.e., lymph sac and EJV), the two cell layers (one of each structure) appear to merge together, forming a single cell layer (Fig. 2E, arrowhead). The most posterior sections show a valve-like opening through which the lymph sac communicates with the veins (Fig. 2F, arrow). There-

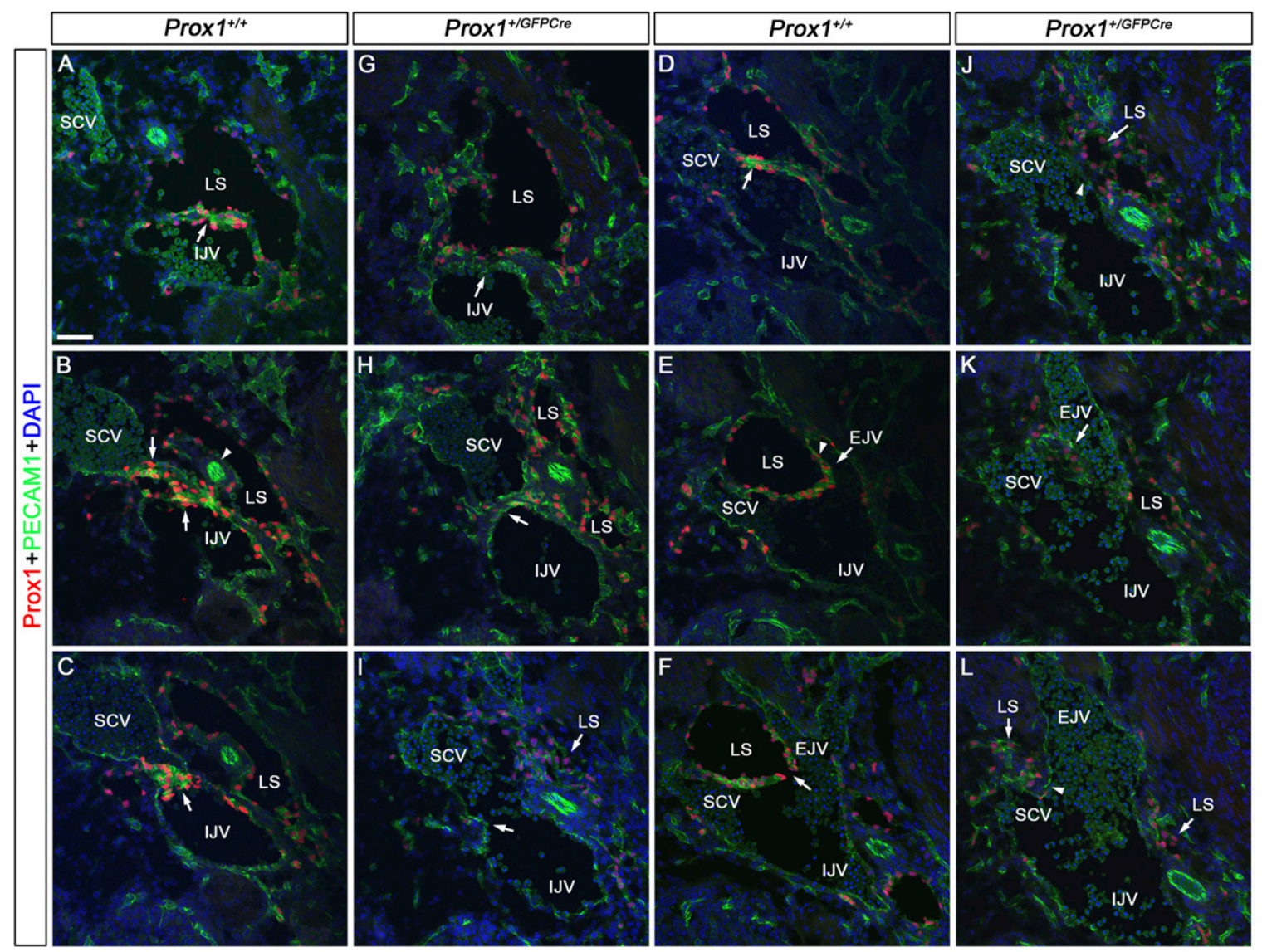

Figure 2. Lymphovenous valves are formed by the fusion of lymph sacs with two adjacent veins. E13.5 wild-type $(A-F)$ or Prox $1^{+/ G F P C r e}(G-L)$ embryos were transversely sectioned in an anterior to posterior orientation in the region where the jugular and subclavian veins interact (box in Fig. 1A) and were immunostained for the LEC marker Proxl and the pan-endothelial marker PECAM1. (A) In wild-type embryos, Proxl is expressed uniformly in LECs forming the lymph sac (LS) and in a polarized manner on the IJV (arrow). Note the relatively high levels of Prox1 on the ECs in the vein and in the lymph sacs' LECs that are facing the vein. $(B)$ The lymph sac is split into two portions by the vertebral artery (white arrowhead). Both walls of the medial portion of the lymph sac intercalate with the wall of the IJV medially and the SCV laterally (arrows). (C) The IIV and the SCV have completely merged together, and the valve rudiment is seen in the middle (arrow). (D) The lateral portion of the lymph sac (LS) runs adjacent to the SCV. Note the relatively higher levels of Proxl in the venous ECs and in the LECs in the lymph sac facing the vein (arrow). (E) The EJV is branching off from the SCV (arrow), and Proxl is expressed on the walls of this vein in a polarized manner (arrowhead). This wall is also adjacent to the lymph sac. (F) The opening of the valve (arrow) is now seen and is formed by the fusion of the two layers of Prox $1^{+}$ECs. $(G, H)$ In Prox $1^{+/ G F P C r e}$ embryos, Proxl is expressed in LECs forming the lymph sac, but very few Prox $1^{+}$cells are seen on the walls of the IJV and SCV (arrows). (I) At the point where the IJV and the SCV merge, no Prox ${ }^{+}$cells or valve-like structures exist (arrow). (/) No Proxl expression was observed on the wall of the SCV that lies close to the lymph sac (arrowhead). (K) Posterior to that, the EJV branches off from the SCV. No Prox $1^{+}$cells are seen on the walls of the EJV (arrow). (L) No communication is observed between the lymph sac and the veins (arrowhead). Also, note an overall reduction in the number of LECs in $G-L$. The neural tube is oriented toward the right, the heart is oriented toward the left, and the thymus is oriented toward the bottom in each panel. Bar, $50 \mu \mathrm{m}$. 
fore, the lymphovenous valves are formed where the junction of two veins (subclavian and internal or external jugular) comes in close contact with the nearby jugular lymph sacs, and these valves are formed by two different types of Prox1-expressing EC: LECs from the lymph sac and a specialized EC type provided by the veins' walls.

\section{Prox1 heterozygous embryos lack lymphovenous valves}

The ECs in the leaflets of the lymphovenous valves expressed high levels of Proxl (Fig. 1B,C, red arrowheads). However, in the lymph sacs, the level of Prox 1 expression among cells was variable, being particularly high in some regions (Fig. 1B,C, yellow arrowheads). To evaluate whether these variable levels of Proxl expression were functionally relevant, we took advantage of two available Prox 1 heterozygous strains (Wigle et al. 1999; Srinivasan et al. 2010). Using antibodies against Prox1, PECAM1, and podoplanin, we first evaluated the junction of the jugular and subclavian veins in frontal sections of E13.5 Prox $1^{+/ G F P C r e}$ embryos. The differences in the levels of Proxl expression among LECs in the lymph sacs of these mutant embryos (Fig. 1D,E) were not as obvious or widespread as in normal controls (Fig. 1B,C). Furthermore, no obvious lymphovenous valve-like structures were evident in Prox1 heterozygous embryos, and venous ECs down-regulated the expression of Proxl (Fig. 1D, arrow). Instead, some Prox1 heterozygous embryos' lymph sacs abruptly fused with veins (Fig. 1E, arrow) and were occasionally blood-filled (data not shown).

Analysis of transverse sections of Prox1 heterozygous embryos at this same region confirmed that Prox 1 is downregulated in the venous walls and that lymphovenous valves are absent (Fig. 2G-L). Additionally, compared with their wild-type littermates, the total number of LECs in Prox $1^{+/ G F P C r e}$ embryos at this stage appears to be reduced; consequently, the lymph sacs were consistently smaller than those of control littermates (Fig. 2).

To confirm whether Prox $1^{+/ G F P C r e}$ embryos were missing valves, we performed additional immunostainings using the LEC markers Vegfr3 (Dumont et al. 1998) and Lyve-1 (Banerii et al. 1999) and the blood EC marker von Willebrand factor (vWF) (Ginsburg et al. 1985). As expected, wild-type control animals had Vegfr3 on lymph sac LECs but not on the venous side (Supplemental Fig. S2A). Lyve-1 was detected on most LECs in the lymph sacs and occasionally on ECs on the venous walls (Supplemental Fig. S2 arrows). In contrast, vWF was detected on venous ECs but not on LECs (Supplemental Fig. S2A). The expression of these markers was similar in Prox $1^{+/ G F P C r e}$ embryos (Supplemental Fig. S2B).

In collecting lymphatics, Foxc2 labels the lymphatic valves, and its activity is crucial for their normal development (Petrova et al. 2004). In the lymphovenous valves of control embryos, Foxc2 expression is restricted to the venous Prox $1^{+}$cells (Fig. 3A, arrow) (occasionally some localized expression was seen also on the LEC side). Integrin- $\alpha 9$ is an additional lymphatic valve marker required for valve development in the collecting lymphatics (Bazigou et al. 2009). This gene was also strongly expressed

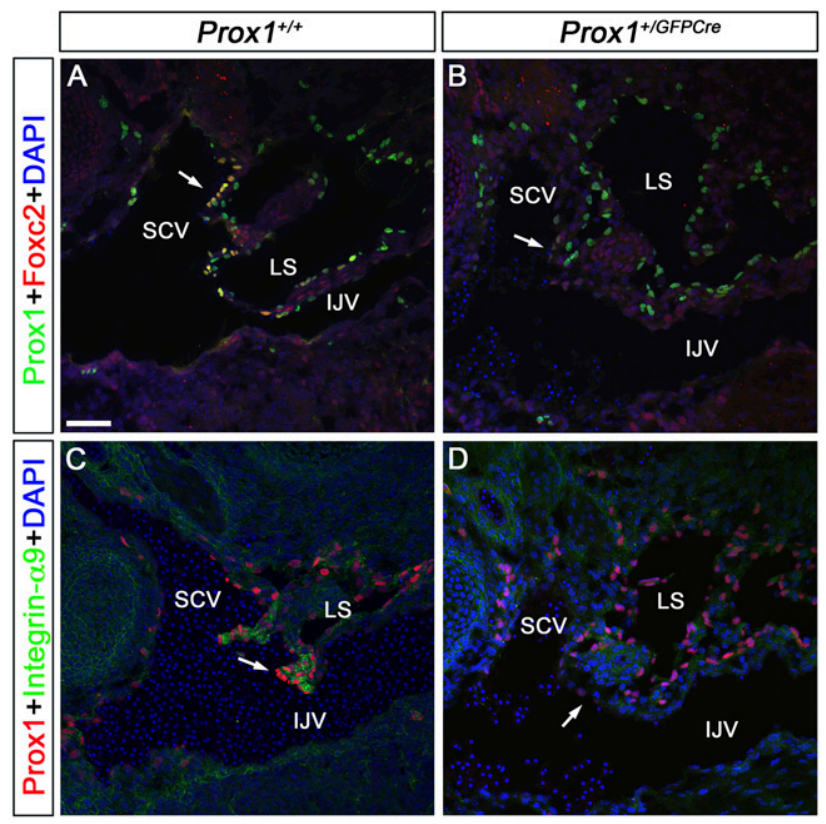

Figure 3. Foxc 2 and Integrin- $\alpha 9$ are expressed in the lymphovenous valves but are absent in Prox1 heterozygous embryos. E13.5 wild-type $(A, C)$ and Prox $1^{+/ G F P C r e}(B, D)$ embryos were frontally sectioned and immunostained for either Prox1 and Foxc2 $(A, B)$ or Prox1 and Integrin- $\alpha 9(C, D)$. $(A)$ In wild-type embryos, Foxc2 is specifically expressed on the outer layer of the valve's leaflets, which are continuous with the walls of the veins (arrow). (B) In sections from an identical region in Prox $1^{+/ G F P C r e}$ embryos, Foxc2 is not expressed on the walls of the veins (arrow). Integrin- $\alpha 9$ is strongly expressed in the valves of control embryos $(C$, arrow) but is absent in Prox $1^{+/ G F P C r e}$ littermates $(D$, arrow). The head is oriented toward the right, the heart is oriented toward the left, and the thymus is oriented toward the bottom in both panels. (IJV) Internal jugular vein; (SCV) subclavian vein; (LS) lymph sac. Bar, $50 \mu \mathrm{m}$.

in the lymphovenous valves of wild-type controls (Fig. 3C, arrow). A similar analysis of Prox $1^{+/ G F P C r e}$ embryos failed to detect Foxc 2 or Integrin- $\alpha 9$ expression in this region, confirming that lymphovenous valves are missing in these embryos (Fig. 3B,D).

\section{The pool of Prox1-expressing venous ECs and LEC progenitors is reduced in Proxl heterozygous embryos}

To better characterize the development of these lymphovenous valves, we compared the expression of Proxl and Foxc2 in frontal sections of E11.5 and E12.5 wild-type control and Prox ${ }^{+/ G F P C r e}$ littermates. At E11.5, only weak Foxc2 expression is observed in Prox $1^{+}$venous ECs (Fig. 4A, arrowhead) and, occasionally, a single valve rudiment starts to be detected at this stage in the CV (future IJV) of control embryos (Fig. 4A, arrow). In contrast, no valves or valve rudiments were present in Prox $1^{+/ G F P C r e}$ embryos (Fig. 4B). We also noticed that the number of Prox $1^{+}$ECs on and outside the CV was reduced in Prox1 heterozygous embryos (Fig. 4C). At E12.5, Foxc $2^{+} /$Prox $^{+}$lymphovenous valve rudiments were evident on the wall of the $\mathrm{CV}$ of control embryos, juxtaposed to Prox $1^{+} /$Foxc $^{-}$lymph sacs (Fig. 4D, arrows). These Foxc $2^{+}$cells were the only Prox $1^{+}$ 


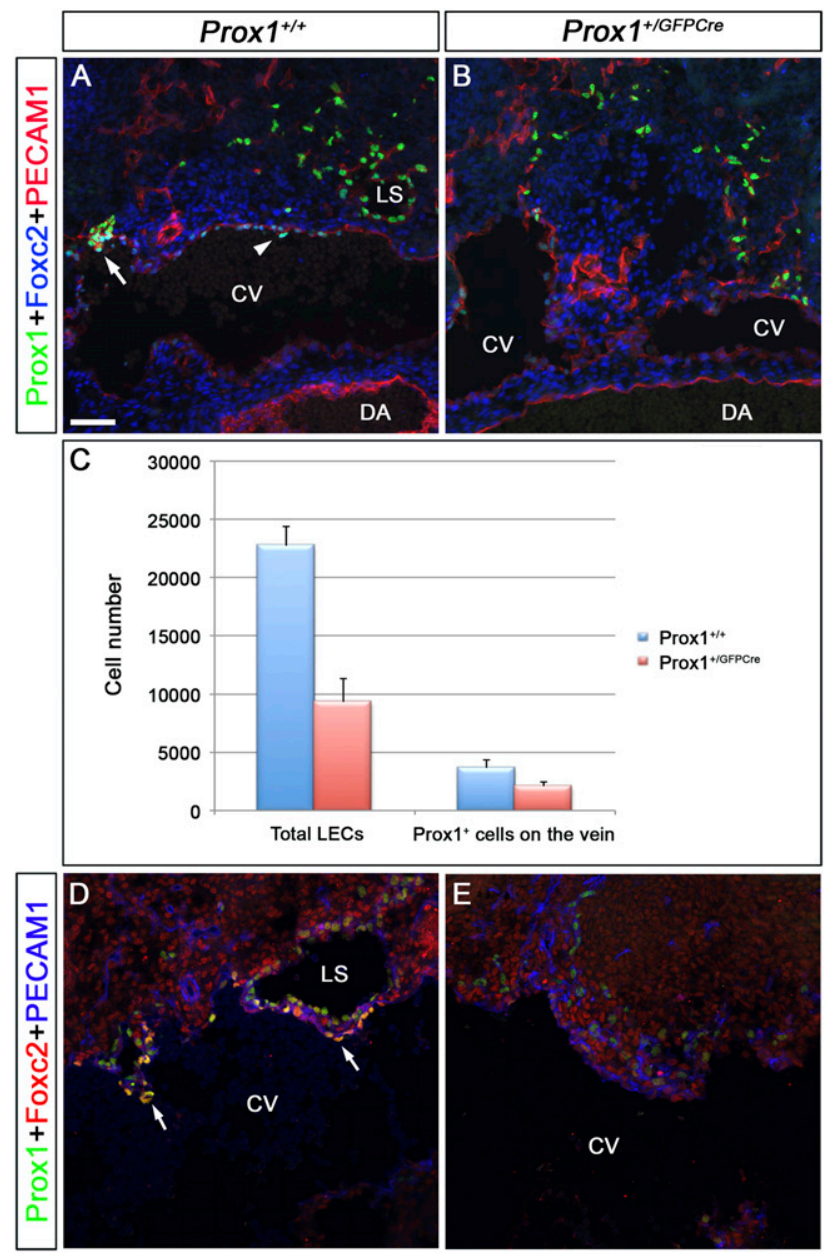

Figure 4. The number of Prox1-expressing LEC progenitors and Prox1-expressing venous ECs that will form the lymphovenous valves is reduced in Prox1 heterozygous embryos. $(A, B)$ E11.5 control $(A)$ and Prox $1^{+/ G F P C r e}(B)$ embryos were frontally sectioned and immunostained for Prox1, Foxc2, and PECAM1. (A) In the control embryos, Foxc2 is weakly expressed in the Prox $1^{+}$ cells on the vein (arrowhead). In addition, lymph sacs and occasionally one valve rudiment (arrow) could be observed. (B) In Prox $1^{+/ G F P C r e}$ embryos, the total number of LECs appears to be reduced, and no lymph sacs or valve rudiments are seen. $(C)$ Compared with wild-type littermates, the total number of Prox $1^{+}$PECAM $1^{+}$ECs on the vein and outside the vein were reduced in E11.5 Prox $1^{+/ G F P C r e}$ embryos $(n=3$ for each genotype, $P<0.05)$. $(D, E)$ E12.5 control $(D)$ and Prox $1^{+/ G F P C r e}(E)$ embryos were frontally sectioned and immunostained for Prox1, Foxc2, and PECAM1. $(D)$ In control embryos, Foxc2 is expressed on the valve rudiments (arrows), which lie adjacent to the primitive lymph sacs. (E) In Prox $1^{+/ G F P C r e}$ embryos, the total number of LECs appears to be reduced, and no valve rudiments are seen. Lymph sacs are also not observed in this location. The head is oriented toward the right, the heart is oriented toward the left, and the thymus is oriented toward the bottom in all panels. (CV) Cardinal vein; (DA) dorsal aorta; (LS) lymph sac. Bar, $50 \mu \mathrm{m}$.

cells on the $\mathrm{CV}$ at this stage. In contrast to that finding but similar to what we found at later stages, no Prox $1^{+} / \mathrm{Foxc}^{+}$ cells were present on the veins of E12.5 Prox $1^{+/ G F P C r e}$ littermates (Fig. 4E), and the total number of LECs outside the veins was also reduced at this stage (Fig. 4D,E). It could be possible that the observed progressive reduction in the number of Proxl-expressing venous ECs in Prox $1^{+/ G F P C r e}$ embryos might be partially responsible for the lack of lymphovenous valves.

To investigate whether the lack of lymphovenous valves could also be due to a developmental delay, we analyzed the expression of Prox1, podoplanin, and PECAM1 in E16.5 Prox $1^{+/ L a c Z}$ embryos. At this stage, the edematous phenotype seen at earlier stages is rescued (Supplemental Fig. S3). Also at this stage, the general appearance of the lymphovenous valves of control embryos is similar to that of those analyzed at E13.5 (Supplemental Fig. S4A, arrow), although the outer Prox $1^{+}$podoplanin ${ }^{-}$cell layer in the leaflets appeared longer (Supplemental Fig. S4A, dotted line); Prox1 expression in venous valves could also be seen at this stage (Supplemental Fig. S4A, arrow; see below). In contrast, in Prox $1^{+/ L a c Z}$ embryos, Prox $1^{+}$cells were rarely seen on venous ECs, Proxl expression remains significantly downregulated in the walls of the IJV, and no valves are present (Supplemental Fig. S4B). In some areas, the lymph sac was abruptly fused with the IJV (Supplemental Fig. S4C); however, only a small number $(<10 \%)$ of Prox1 heterozygous embryos had blood-filled superficial lymphatics (Supplemental Fig. S3). This finding suggests that additional alterations are required for blood to breach the lymphatic vasculature. Occasionally, we also saw lymphatic vessels from the para-tracheal lymph plexus opening into the pleural cavity of Prox1 heterozygous embryos (Supplemental Fig. S4D, arrow), a defect that could be partially responsible for the chylothorax (i.e., accumulation of chyle in the thoracic cavity) seen in newborn Prox1 heterozygous pups (Harvey et al. 2005). Taken together, our findings show that Prox 1 activity is required for the proper formation of the lymphovenous valves in a dose-dependent manner; therefore, these valves are defective in Prox1 heterozygous embryos with abnormal lymphovenous connections. These abnormal connections are likely used to relieve the excessive pressure due to fluid accumulation in the lymphatic vessels.

\section{The lack of lymphovenous valves in Prox 1 heterozygous embryos is caused by defective maintenance of venous Proxl expression}

It was previously suggested that two steps are necessary during the initial LEC differentiation process: an early Prox1 initiation step dependent on Coup-TFII and Sox18, and a later Prox1 maintenance step dependent on Prox1 itself (Francois et al. 2008; Srinivasan et al. 2010). Our results showing that losing a copy of Prox 1 triggers a reduction in the number of Prox $1^{+}$cells in the veins without affecting their rate of proliferation or cell death (data not shown) suggested that the Prox1-dependent maintenance step could be affected in these heterozygous embryos. To evaluate this possibility, we performed lineage-tracing analysis by crossing Prox $1^{+/ C r e E R T 2}, R 26 R^{+/ Y F P}$ mice (Srinivas et al. 2001; Srinivasan et al. 2007) with $\operatorname{Prox} 1^{+/ L a c Z}$ mice (Wigle et al. 1999) and injecting the pregnant females with $2 \mathrm{mg}$ of tamoxifen per $40 \mathrm{~g}$ of body weight at E10.5. Prox $1^{+/ C r e E R T 2} ; R_{26 R^{+/ Y F P}}$ and Prox $1^{\text {LacZ/CreERT2 }} ; R_{2} 6 R^{+/ Y F P}$ 
embryos isolated from those crosses were analyzed at E13.5. Using this approach, LEC progenitors should be labeled as soon as Proxl expression is initiated /around E10.0). In this case, if Proxl maintenance is normal, all labeled $\mathrm{YFP}^{+}$ECs also will be Prox ${ }^{+}$; however, if Prox1 maintenance is defective, those ECs will be Prox $1^{-} \mathrm{YFP}^{+}$.

As described before, numerous Prox $1^{+}$cells were detected in the lymphovenous valve region of control PrOX $1^{+/ C r e E R T 2} ; R 26 R^{+/ Y F P}$ embryos, and some of these coexpressed YFP, a result indicating that that these cells are descendants of LEC progenitors that were present at E10.5 (Supplemental Fig. S5A,B). Importantly, Prox ${ }^{-}$YFP $^{+}$cells were not detected in these embryos (Supplemental Fig. S5A,B). Instead, Prox1 expression was down-regulated in the putative lymphovenous valve territory of Prox1 heterozygous (Prox $1^{\text {LacZ/CreERT2 }}$,R26R ${ }^{+/ Y F P}$ ) littermates (Supplemental Fig. S5C), although few $\mathrm{YFP}^{+}$cells were detected in this region (Supplemental Fig. S5D, arrow). Therefore, these $\mathrm{YFP}^{+}$cells correspond to the original LEC progenitors that failed to maintain Proxl expression and thus were unable to differentiate into valve cells.
PrOx $1^{+/ C r e E R T 2}$-mediated labeling is mosaic and occurs only during the short developmental time frame when tamoxifen is active; therefore, we performed a similar analysis in E13.5 Prox ${ }^{+/ G F P C r e} ; R 26 R^{+/ Y F P}$ embryos. Likewise, Prox1 expression was down-regulated; however, numerous $\mathrm{YFP}^{+}$cells were present in the putative lymphovenous valve region (Fig. 5A, arrows). As indicated before, some Prox 1 heterozygous embryos have abnormal lymphovenous connections and blood-filled lymphatics at this stage (Fig. 5B, arrow). We also detected several Prox $^{-} / \mathrm{YFP}^{+}$cells in the IJV in this area (Fig. 5B, arrowheads). These observations suggest that in Prox1 heterozygous embryos there is a defect in the maintenance of Prox 1 expression in LECs and lymphovenous valve progenitors.

\section{CoupTFII/Prox1 complex formation is reduced in Proxl heterozygous LEC progenitors}

Next, we aimed to identify the mechanisms leading to the silencing of Prox1 expression in Prox1 heterozygous embryos. As mentioned above, two steps are likely involved
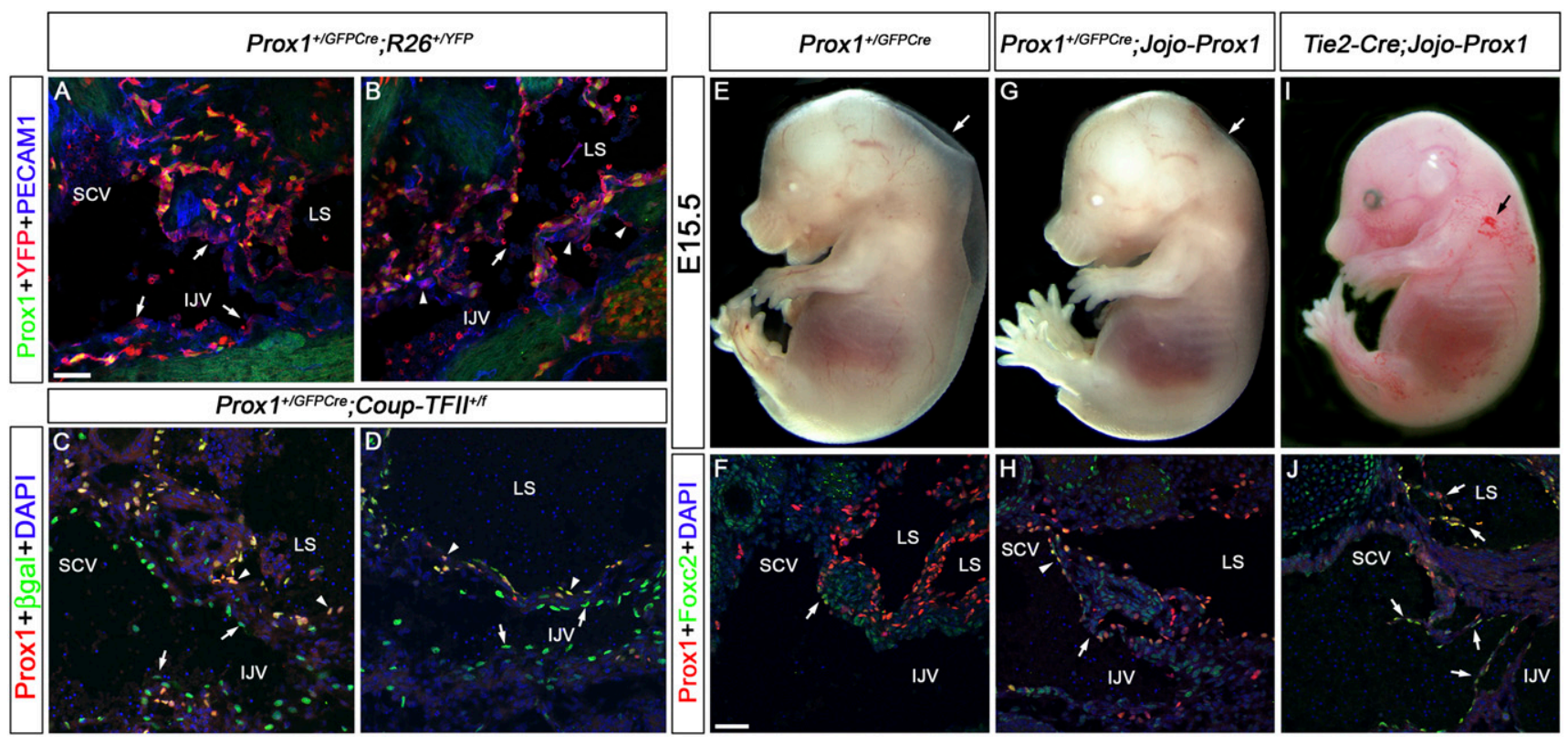

Figure 5. Prox1 heterozygous embryos do not maintain Prox 1 expression in a subpopulation of LEC and lymphovenous valve progenitors. $(A, B)$ E13.5 Prox $1^{+/ G F P C r e} ; R_{26}{ }^{+/ Y F P}$ embryos were sectioned and immunostained for Prox 1, YFP, and PECAM1. (A) No Prox $1^{+}$ECs are seen in the area that should have developed into the lymphovenous valve; however, numerous $\mathrm{YFP}^{+}$cells can be detected (arrows). (B) In some Prox1 heterozygous embryos that develop blood-filled lymphatics at this stage, abnormal connections between the lymph sacs and the IJV are observed (arrow); several Prox $1^{-} \mathrm{YFP}^{+}$cells are seen in this area (arrowheads). The $\mathrm{YFP}^{+} \mathrm{PECAM1}^{-}$cells in the lumen of the blood vessel and the lymph sacs are hematopoietic cells. $(C, D)$ E15.5 Prox $1^{+/ G F P C r e}$;Coup-TFII ${ }^{+/ f}$ embryos were immunostained for Proxl and $\beta$-galactosidase. $(C, D)$ The blood-filled lymph sac is clearly seen and is lined by Prox $1^{+} \beta$-gal ${ }^{+}$LECs (arrowheads). $(C)$ No Proxl expression is seen on veins in the region where the valves are normally formed (arrows), but $\beta$-gal ${ }^{+}$cells are detected in this region. $(D)$ Proxl $^{-} \beta$-gal ${ }^{+}$ cells line the IIV (arrows). (E-I) Jojo-Prox1 mice were bred to Prox $1^{+/ G F P C r e}$ or Tie2-Cre mice and the resulting embryos were analyzed at E15.5 by whole mount or by immunohistochemistry on frontal sections with antibodies against Prox 1 and Foxc 2 . Prox $1^{+/ G F P C r e}$ embryos display edema ( $E$, arrow), and the expression of Prox1 and Foxc2 is reduced in the region where the valves are normally formed $(F$, arrow). $(G)$ Expression of Prox1 in Prox $1^{+/ G F P C r e}$; Iojo-Prox1 embryos rescues the lymphatic vascular phenotype, as these embryos display no obvious edema (arrow). (H) Expression of Prox1 and Foxc2 in the venous ECs (arrowhead) and the lymphovenous valves (arrow) is observed in Prox ${ }^{+/ G F P C r e}$;IOjo-Prox1 embryos. (I) Misexpression of Proxl in blood ECs in Tie2-Cre; Iojo-Prox1 embryos results in a blood-filled lymphatic phenotype (arrow). (I) Numerous abnormal valve-like structures composed of Prox $1^{+}$Foxc2 ${ }^{+}$cells were observed in the IJV and lymph sac (LS) of Tie2-Cre; Iojo-Prox1 embryos (arrows). In the sections, the head is oriented toward the right, the heart is oriented toward the left, and the thymus is oriented toward the bottom in all panels. (IJV) Internal jugular vein; (SCV) subclavian vein; (LS) lymph sac. Bar, $50 \mu \mathrm{m}$. 
in regulating Prox1 expression (Srinivasan et al. 2010). The Prox1-dependent maintenance step, which occurs when Prox 1 expression is induced and LEC differentiation is progressing, can be further subdivided into an early Coup-TFII/Prox1-dependent step that occurs on the vein and a later Coup-TFII-independent step that occurs when LECs move out from the vein (Srinivasan et al. 2010). The observed reduction in Proxl expression in the veins of Prox1 heterozygous embryos could be caused by defects in the maintenance step (i.e., the Coup-TFII/Prox1-dependent step). Directly quantifying the amount of CoupTFII/Prox 1 complex is technically difficult because there are few LEC progenitors in Prox1 heterozygous embryos, there are no available surface markers to sort these cells, and there are two different Prox $1^{+}$cell populations on the vein at any given time (those that will maintain and those that will not maintain Proxl expression). Therefore, as an alternative method to study the Coup-TFI/Proxl complex, we used an algorithmic approach. According to Langmuir isotherm (Copeland 2000), Coup-TFII/Proxl interaction could be represented as a bimolecular reaction as follows: [Coup-TFII] + [Prox1] $\rightarrow$ [Coup-TFII/Prox1] (brackets represent the equilibrium concentrations). The amount of Coup-TFII/Proxl complex could be represented as [Coup-TFII/Prox1] $=K_{a}$ [Coup-TFII][Prox 1$]$, where $k_{a}$ is the equilibrium association constant and is dependent on the intrinsic characteristics of the interacting proteins (i.e., the strength of electrostatic and hydrophobic interactions and extrinsic properties such as temperature, osmolarity, and $\mathrm{pH}$ ).

By considering $k_{a}$ and [Coup-TFII] to be the same, it is easy to appreciate that the amount of Coup-TFII/Prox1 complex formed in Prox1 heterozygous LECs will be half that formed in wild-type LECs. We hypothesized that this reduction in the amount of Coup-TFI/Prox1 complex formed could be the reason for the reduction in Prox 1 expression. Then, if Coup-TFII levels are reduced in addition to those of Prox1, the amount of Coup-TFII/Prox1 complex formed should be further reduced, and the resulting phenotype should be more severe. We used Prox $1^{+/ G F P C r e}$ mice that were bred with Coup-TFII ${ }^{+/ f}$ mice to generate double-heterozygous animals; most of those pups die at birth in mixed backgrounds or have a significantly low survival $(2.5 \%)$ in the NMRI background (Srinivasan et al. 2010). Accordingly, we collected double-heterozygous embryos at either E11.5 or E15.5. At E11.5, significantly fewer Prox $1^{+}$cells were on or outside the veins of Prox $1^{+/ G F P C r e}$; Coup-TFII ${ }^{+/ f}$ embryos compared with their Prox $1^{+/ G F P C r e}$ littermates (Supplemental Fig. S6A). At E15.5, all of the double-heterozygous embryos had severe edema and variable blood-filled lymphatics (Supplemental Fig. S6B-E). Lineage-tracing analysis using the $L a c Z$ reporter that is activated when the Coup-TFII allele is deleted (Takamoto et al. 2005) showed that the lymphovenous valves were absent in the double-heterozygous embryos and were replaced by Prox1 ${ }^{-} \beta_{\text {gal }}{ }^{+}$cells as expected (Fig. 5C, arrows). Additionally, we observed numerous Prox ${ }^{-} \beta$ gal $^{+}$cells lining the IJV, indicating a dramatic reduction in the number of differentiating LECs (Fig. 5D, arrows). In contrast, in E13.5 Prox $1^{+/ C r e E R T 2}$; Coup-TFII ${ }^{+/ f}$ embryos exposed to tamox- ifen at E10.5, no Prox $1^{-} \beta$ gal $^{+}$ECs were seen in the vein (Supplmental Fig. S7A,B).

To confirm that LECs is the original fate of the labeled cells $\left(\right.$ Prox $^{-} / \mathrm{YFP}^{+}$cells in Prox $1^{+/ G F P C r e} ; R 26 R^{+/ Y F P}$ embryos and Prox $1^{-} / \beta$-gal ${ }^{+}$cells in Prox $1^{+/ \text {GFPCre }} ;$ Coup-TFII ${ }^{+/ f}$ embryos) and that they do not arise because of aberrant or leaky expression of the Prox $1^{+/ G F P C r e}$ line, we performed a rescue experiment using a previously reported Prox1 conditional transgenic line (Lavado et al. 2010). This conditional gain-of-function strain (Jojo-Prox1) will express Proxl only in cells exposed to Cre recombinase. We then crossed this transgenic strain with the Prox $1^{+/ G F P C r e}$ strain and observed that in contrast to their Prox $1^{+/ G F P C r e}$ littermates (Fig. 5E, arrow), E15.5 double-heterozygous embryos were completely devoid of edema (Fig. 5G, arrow). Immunostainings of frontal sections of Prox $1^{+/ G F P C r e}$; Jojo-Prox 1 embryos against Proxl and Foxc2 confirmed that the expression of these two markers as well as the lack of the lymphovenous valves were rescued in these embryos (Fig. 5F,H). In contrast, double-heterozygous embryos generated by crossing the Prox1 transgenic strain with Tie2-Cre (Kisanuki et al. 2001) that express Prox1 in blood ECs display blood filled lymphatics (Fig. 5I) and ectopic abnormally shaped valves within the vein and the lymph sac (Fig. 5J, arrows). This result confirmed that the original fate of the labeled Prox $1^{-}$cells is to be LECs and demonstrated that Prox1 heterozygous embryos have a defect in the proper maintenance of Prox 1 expression, resulting in the loss of LEC progenitors and lymphovenous valves.

Next, we studied the contribution of $k_{a}$ and [CoupTFII] to the reaction kinetics. To determine the role of $k_{a}$, we used previously generated $\operatorname{Prox} 1^{+/ N R}$ mice in which the nuclear hormone interaction motif (and therefore the Coup-TFII interaction motif) is mutated (Srinivasan et al. 2010). Although mutating the nuclear hormone interaction motif affects the $k_{a}$ between CoupTFII and Prox1 (Srinivasan et al. 2010), Prox $1^{+/ N R}$ embryos are normal (Srinivasan et al. 2010), and the development of the valves is not affected in these mutant embryos (Fig. 6A,B). Therefore, the reduction in $k_{a}$ caused by the mutation in the nuclear hormone interaction motif is not sufficient to cause a valve defect.

We then investigated the role of [Coup-TFII] in the formation of the valves in Tie2-Cre;Coup-TFII ${ }^{+/ f}$ embryos. A small number of Tie2-Cre;Coup-TFII ${ }^{+/ f}$ embryos had mild edema (data not shown); otherwise, these EC-specific CoupTFII heterozygous embryos were normal (Fig. 6C) and their lymphovenous valves were indistinguishable from those of wild-type controls (Fig. 6D). However, a blood-filled lymphatics phenotype and lack of lymphovenous valves were observed in E13.5 Prox ${ }^{+/ C r e E R T 2}$;Coup-TFIf ${ }^{f / f}$ embryos exposed to tamoxifen at E10.5 (Supplemental Fig. S7C).

Next we tested the additive contribution of $k_{a}$ and [Coup-TFII] in Tie2-Cre;Coup-TFII ${ }^{+/ f} ;$ Prox $1^{+/ N R}$ embryos and pups. In contrast to single-heterozygous animals, these compound-mutant animals were indistinguishable from Prox1 heterozygous mice, as they had severe edema at E13.5 (data not shown) and at E15.5 (Fig. 6E) and lacked lymphovenous valves (Fig. 6F, arrows). Furthermore, sim- 


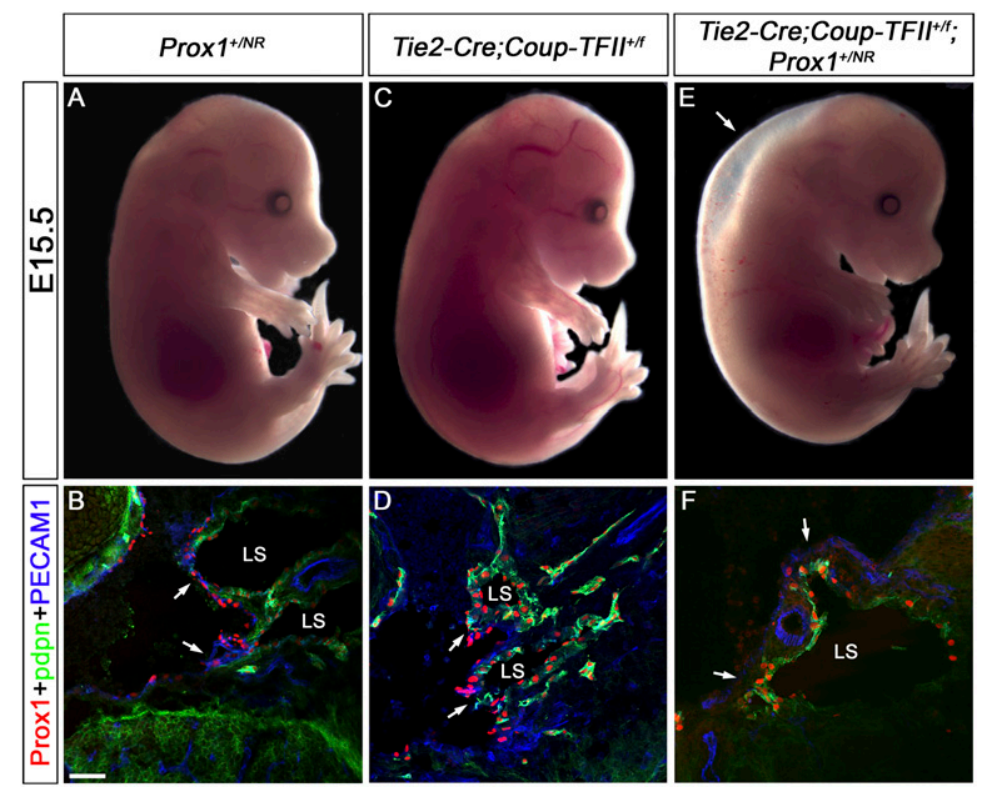

Figure 6. Proxl haploinsufficiency is the result of a reduction in Coup-TFII/Prox 1 interaction. Prox $1^{+/ N R}$ and Tie2-Cre;Coup-TFII ${ }^{+/ f}$ mice were bred and the resulting embryos were analyzed at E15.5 by whole mount or by immunohistochemistry on frontal sections with antibodies against Prox1, podoplanin (pdpn), and PECAM1. $(A-D)$ Prox $1^{+/ N R}$ and Tie2-Cre;Coup-TFII $I^{+/ f}$ embryos are phenotypically normal with unremarkable valves. Instead, Tie2-Cre;Coup-TFII ${ }^{+/ f}$; Prox $1^{+/ N R}$ embryos are edematous $\left(E\right.$, arrow), devoid of Prox $1^{+}$cells in the veins $(F$, arrows), and lack lymphovenous valves. In the sections, the head is oriented toward the right, the heart is oriented toward the left, and the thymus is oriented toward the bottom in all panels. (LS) Lymph sac. Bar, $50 \mu \mathrm{m}$. ilar to Prox1 heterozygotes, compound mutants also had chylothorax, a reduced survival rate after birth (data not shown), and a reduced number of Prox $1^{+}$ECs on or outside the vein at E11.5 (Supplemental Fig. S8). A less severe reduction in the number of Prox $1^{+}$cells was also observed on or outside the veins of E11.5 Prox $1^{+/ N R}$ and Tie2Cre;Coup-TFII ${ }^{+/ f}$ embryos (Supplemental Fig. S8), a result that could explain the lack of any obvious lymphatic defects in these mutant embryos. These data further suggests that the lack of lymphovenous valves is likely due to a reduction in the amount of Coup-TFII/Prox1 complex that forms during LEC specification.

\section{Prox1 dosage is also important for the formation of venous valves}

Valves are important for the normal physiology of the cardiovascular system and are also present in collecting lymphatic vessels, heart, and veins. Prox 1 expression was previously reported in cardiac valves (Risebro et al. 2009) and in collecting lymphatic vessels (Bazigou et al. 2009; Norrmen et al. 2009), but we did not observe any obvious defect in the development of these valves in Prox1 heterozygous embryos (Fig. 7A-C; Supplemental Fig. S9). We decided to analyze whether Proxl is expressed in venous valves and whether the development of these valves is defective in Prox1 heterozygous embryos. Venous valves develop later than the lymphovenous valves and can be seen at the junction of jugular and subclavian veins guarding their entry point (Kampmeier 1928). Accordingly, we found that at E16.5, the venous valves are also Prox $1^{+}$(Fig. $7 \mathrm{D}$, arrowheads). In contrast, similar to the lymphovenous valves, no venous valves were detected in Prox 1 heterozygous embryos (Fig. 7E) at this stage, although few Prox $1^{+}$cells were present on the veins.

A possible explanation for the lack of venous and lymphovenous valves in Proxl heterozygous embryos could be that they have a common origin. To test this possibil- ity, we generated E16.5 Prox $1^{+/ C r e E R T 2} ; R 26 R^{+/ L a c Z}$ embryos and exposed them to tamoxifen $(5 \mathrm{mg}$ per $40 \mathrm{~g}$ of body weight of pregnant dams) at E10.5, a stage when LECs and lymphovenous valve progenitors arise. As expected, we observed several labeled cells in the lymphovenous valves (Fig. 7F, arrows); however, few, if any, labeled cells were observed in the venous valves (Fig. 7F, arrowheads), a result indicating that the venous valves originate later than LECs and lymphovenous valves. The few Prox $1^{+}$ cells observed in the veins of E13.5 embryos away from the lymphovenous valves (Fig. 1B,C, green arrowheads) are the likely source of these valves.

\section{Discussion}

"The duct lies for some distance against the vein, the two being separated only by a double layer of endothelium, one for the vein and one for the lymph duct. Finally, in each series, one can see that, just at the edge of the lymph duct, these two layers are continuous" (Sabin 1902).

Almost a century after the existence of lymphovenous valves at the junction of the jugular lymph sacs with the jugular and subclavian veins was first reported, our work provides a detailed molecular framework for Sabin's accurate anatomical description (Sabin 1902), showing that, indeed, there is a gradual merger of the ECs of the lymph sacs with the walls of the two adjacent veins (Supplemental Fig. S10). Thus, the lymph sac merges at a site where two veins branch off, effectively converting the branch point into a valve.

We previously suggested that the formation of the lymphatic vasculature is a stepwise process that starts with the expression of Prox 1 in a subpopulation of venous ECs (Oliver and Harvey 2002). The new data presented in this study indicate that although the majority of those Prox1expressing ECs (LEC progenitors) will eventually move out from the veins, acquire the expression of additional 


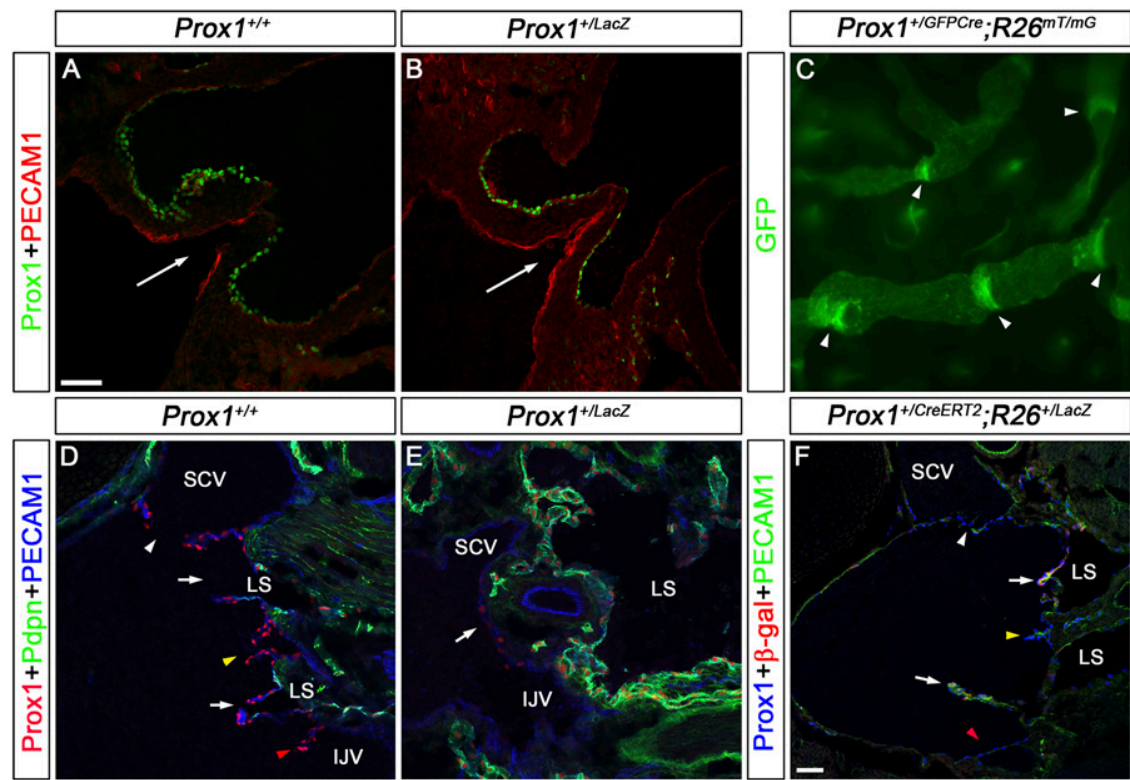

Figure 7. Prox1 heterozygous embryos have defective venous valves. $(A, B)$ The semilunar valve in the outflow tract from the right ventricle was analyzed in E16.5 wild-type $(A)$ and Proxl heterozygous $(B)$ embryos using Proxl and PECAM1 antibodies. Arrows indicate the direction of blood flow. In both embryos, Prox1 is expressed on the downstream side of the valves and no obvious defect was observed in the morphology of the valves. $(C)$ The lymphatic vessels in the ears of adult Prox $1^{+/ G F P C r e} ; R 26 R^{m T / m G}$ mice were analyzed by the autofluorescence of the membrane tagged GFP activated by Cre expression. The valves in the lymphatic vessels could be seen clearly and appear normal. $(D, E)$ On frontal sections, the venous valves were analyzed by immunostaining for Prox1, PECAM1, and podoplanin (Pdpn) in E16.5 wild-type (D) and Prox1 heterozygous $(E)$ embryos at the junction of the jugular and subclavian veins. $(D)$ In control embryos, the venous valves could be seen at the outlets of the SCV (white arrowhead), EJV (yellow arrowhead), and IJV (red arrowhead). The lymphovenous valves are also seen (arrows). (E) In contrast, in Prox1 heterozygous embryos, few Prox $1^{+}$cells are seen on the veins (arrow) but no valve is detected. (F) PrOx $1^{+/ C r e E R T 2}$ and $R 26 R^{+/ L a c Z}$ mice were bred and the pregnant mice were exposed to tamoxifen at E10.5, a stage when LECs are specified. The lymphovenous valves were subsequently analyzed at E16.5 on frontal sections by immunostaining for Prox1, PECAM1, and $\beta$-gal. While $\beta$-gal ${ }^{+}$cells could be observed on the lymphovenous valves (arrows), hardly any labeled cells were observed in the venous valves guarding the subclavian (white arrowhead), external jugular (yellow arrowhead), and internal jugular (red arrowhead) veins, indicating a later origin of these cells. The head is oriented toward the right of $A, B$, and $D-F$. (IJV) Internal jugular vein; (SCV) subclavian vein; (LS) lymph sac. $A-E$ have the same image magnification. Bar, $50 \mu \mathrm{m}$.

LEC markers (LEC specification), and further differentiate, a smaller subpopulation will remain in the veins and help form the lymphovenous valves. This finding also indicates that Proxl-expressing venous ECs represent the pool of common progenitors that give rise to both LECs and lymphovenous valves. In the case of Prox1 heterozygous embryos, the original number of Proxl-expressing venous ECs is reduced. This alteration leads to a reduction in the number of LEC progenitors and the lack of lymphovenous valves. Most likely, whether a Proxl-expressing EC becomes an LEC or a lymphovenous valve EC is determined by the process that regulates the exit of LEC progenitors from the veins; i.e., only those ECs that move out from the veins will become fully differentiated LECs.

One interesting question is why lymphovenous and venous valves, but not cardiac and collecting lymphatic valves, are dependent on Proxl dosage. One possible explanation could be that, as we previously showed, CoupTFII is dispensable in fully differentiated LECs after E13.5 (Srinivasan et al. 2010), and, as collecting lymphatic valves develop after E16.5 (Norrmen et al. 2009), they are probably under the control of a different genetic program. Consistent with this proposal, we were unable to observe any significant Coup-TFII expression in the cardiac valve, which also develops normally in Prox1 heterozygous embryos (Supplemental Fig. S11). Alternatively, blood flow is known to regulate endothelial gene expression (Dekker et al. 2002), and the valves in collecting lymphatics are not exposed to blood flow. Interestingly, in the cardiac valve, Prox1 is ex- pressed opposite to the flow side in a perfectly complementary pattern to that of Klf2, a flow-regulated gene (Lee et al. 2006). The above observation suggests that Prox 1 could be negatively regulated by shear force of blood flow. Consistent with this proposal, the lymphovenous and venous valves are directly exposed to blood flow and might require two copies of Prox 1 for their normal development and maintenance.

Our finding that the lymphovenous valve needed to connect the lymphatic and blood vasculatures is missing in Prox1 heterozygous embryos, allowing lymph sacs to abruptly merge with nearby blood vessels or the pleural cavity, might partially explain the abnormal lymphangiography patterns observed in adult Proxl heterozygous mice (Harvey et al. 2005). Additionally, the importance of this valve in efficiently regulating the return of lymph fluid to the blood circulation and in preventing blood from entering the lymphatic vasculature is evident from our characterization of Prox1 heterozygous embryos, a percentage of which had a blood-filled lymphatics phenotype. The total number of LECs could be the crucial determinant of whether blood can breach the lymph sacs, as we observed a significant reduction in the number of these cells in Prox $1^{+/ G F P C r e}$;Coup-TFII ${ }^{+/ f}$ embryos that displayed blood-filled lymphatics. This phenotype resembles the one previously described in Syk-, Slp-76-, and podoplanin-null embryos (Abtahian et al. 2003; Uhrin et al. 2010). These molecules have been shown to play an important role in regulating LEC-platelet interaction 
during lymphatic-blood separation. It could be possible that somehow these molecules also regulate the number of Prox1-expressing venous EC and LEC progenitors. In this case, we will assume that lymphovenous valves should also be absent in these mutant embryos.

We previously reported that there are two phases of Prox1 regulation in LECs: an early initiation step dependent on Coup-TFII and Sox18, and a later maintenance step dependent on Proxl autoregulation (Srinivasan et al. 2010). Here we show that this autoregulation of Prox 1 expression is dose-dependent and that the total number of Prox1-expressing progenitors in the vein of Prox 1 heterozygous mice is reduced because the amount of available Coup-TFII/Proxl protein complex during the early stages of Prox1 regulation is reduced. This may be because, although the concentration of Coup-TFII is at steady-state levels during LEC specification, Proxl is just beginning to be expressed during this stage and is therefore the only true variable involved in this protein-protein interaction kinetics. This, in combination with a weak residual interaction between the mutated Proxl and Coup-TFII, could be the reason for the milder phenotype observed in Prox $1^{+/ N R}$ and Tie2-Cre;Coup-TFII $I^{+/ f}$ compared with that of Tie2Cre;Coup-TFII ${ }^{+/ f}$; Prox $1^{+/ N R}$ embryos. It is possible that some transcription factor may compete with Coup-TFII/ Prox 1 complexes in a dose-dependent manner to negatively regulate Prox 1 expression and thereby control the number of LEC progenitors that are generated in the veins. This scenario would be similar to that which occurs during angiogenesis when the correct number of tip versus stalk cells is determined by the Notch signaling activator VEGF-A and its inhibitor, Dll4 (Hellstrom et al. 2007). In the case of $V E G F-A$ and D114, this balance goes awry in embryos heterozygous for either of these genes, indicating the importance of a delicate balance between two opposing pathways in this process (Hellstrom et al. 2007). Our results highlight a similar role for Proxl in determining lymphatic versus blood EC fates in a concentration-dependent manner during developmental lymphangiogenesis. Identifying and characterizing Proxl's blood EC counterpart will provide further insight into the mechanisms that precisely control the blood EC versus LEC differentiation process.

In conclusion, we now provide a detailed molecular framework to understand the formation of the lymphovenous valves. Characterizing these valves in patients with primary lymphedema or other lymphatic alterations should offer further insight into the function of these structures in health and disease.

\section{Materials and methods}

Mice

The generation of $R 26 R^{+/ L a c Z}$, Prox $1^{+/ L a c Z}$, Tie2-Cre, $R 26 R^{+/ Y F P}$, Coup-TFII ${ }^{+/ f}, R 26 R^{m T / m G}$, Prox $^{+/ C r e E R T 2,}$, Jojo-Prox1,Prox $1^{+/ N R}{ }^{\prime}$, and Prox $1^{+/ G F P C r e}$ mutant mice has been described (Soriano 1999; Wigle et al. 1999; Kisanuki et al. 2001; Srinivas et al. 2001; Takamoto et al. 2005; Muzumdar et al. 2007; Srinivasan et al. 2007, 2010; Lavado et al. 2010). All described mouse experiments were approved by the St. Jude Children's Research Hospital Animal Care and Use Committee.

\section{Antibodies}

The primary antibodies used were rabbit anti-Proxl (AngioBio), goat anti-Prox 1 (R\&D Systems), rat anti-PECAM1 (BD Pharmingen), rabbit anti-GFP (Molecular Probes), guinea pig anti-Lyve-1 (in-house), hamster anti-podoplanin (Hybridoma Bank), goat anti-Vegfr3 (R\&D Systems), rabbit anti-vWF (DAKO), goat antiIntegrin- $\alpha 9$ (R\&D Systems), and rabbit anti-Foxc2 (in-house). The secondary antibodies used were $\mathrm{Cy} 3$-conjugated donkey anti-rabbit (Jackson ImmunoResearch Laboratories), Cy3-conjugated donkey anti-rat (Jackson ImmunoResearch Laboratories), Alexa 488conjugated donkey anti-rat (Molecular Probes), Alexa 488-conjugated goat anti-hamster (Molecular Probes), Alexa 488-conjugated donkey anti-goat (Molecular Probes), Dylight 649-conjugated donkey anti-rabbit (Jackson ImmunoResearch Laboratories), DyLight 649-conjugated donkey anti-guinea pig (Jackson ImmunoResearch Laboratories), and Cy5-conjugated anti-rat (Jackson ImmunoResearch Laboratories).

\section{Tamoxifen administration}

Tamoxifen preparation and intraperitoneal injections of pregnant dams were performed as previously described (Srinivasan et al. 2007).

\section{Immunohistochemistry}

Fluorescent immunohistochemistry, X-gal staining, and horseradish peroxidase staining using 3,3'-diaminobenzidene (DAB) as a substrate were performed as previously described (Srinivasan et al. 2007). The sections were mounted by using mounting medium containing DAPI (Vectashield), and confocal microscopy was performed as described previously (Srinivasan et al. 2010). To image the valves in the lymphatic vessels of the ear, adult wild-type and Prox ${ }^{+/ G F P C r e} ; R 26 R^{m T / m G}$ mice were perfused and the ears were collected and processed for whole mount as described previously (Harvey et al. 2005). Subsequently, wildtype ears were immunostained with an antibody specific for podoplanin, whereas the Prox $1^{+/ G F P C r e} ; R 26 R^{m T / m G}$ ears were visualized directly using the autofluorescence of the activated GFP reporter (Muzumdar et al. 2007). To image the valves in the collecting lymphatic vessels of the mesentery, guts of newborn $R 26 R^{m T / m G}$ and Prox $1^{+/ G F P C r e} ; R 26 R^{m T / m G}$ pups were harvested and whole-mount immunohistochemistry was performed for Foxc2 or Prox1.

\section{Statistical analysis}

Microsoft Excel was used to evaluate the statistical significance by unpaired Student's $t$-test.

\section{Acknowledgments}

We thank Drs. S. Tsai and M.-J. Tsai for providing the Coup-TFII floxed mouse strain. We thank Xin Geng and Betsy Willford for help with the figures; Ashley Interiano for help with cell counting; Hari Kamadurai for critical reading of the manuscript; Samuel Connell, Yannan Ouyang, and Jennifer Peterson for help with confocal imaging; and Cherise Guess for editing the manuscript. We thank all the members of our laboratory for their helpful suggestions and discussions. This work was supported by NIH grant R01- 
HL073402 (to G.O.) and by the American Lebanese Syrian Associated Charities (ALSAC).

\section{References}

Abtahian F, Guerriero A, Sebzda E, Lu MM, Zhou R, Mocsai A, Myers EE, Huang B, Jackson DG, Ferrari VA, et al. 2003. Regulation of blood and lymphatic vascular separation by signaling proteins SLP-76 and Syk. Science 299: 247251.

Banerji S, Ni J, Wang SX, Clasper S, Su J, Tammi R, Jones M, Jackson DG. 1999. LYVE-1, a new homologue of the CD44 glycoprotein, is a lymph-specific receptor for hyaluronan. J Cell Biol 144: 789-801.

Bazigou E, Xie S, Chen C, Weston A, Miura N, Sorokin L, Adams $\mathrm{R}$, Muro AF, Sheppard D, Makinen T. 2009. Integrin- $\alpha 9$ is required for fibronectin matrix assembly during lymphatic valve morphogenesis. Dev Cell 17: 175-186.

Copeland RA. 2000. Enzymes: a practical introduction to structure, mechanism and data analysis. Wiley-VCH, New York.

Dekker RJ, van Soest S, Fontijn RD, Salamanca S, de Groot PG, VanBavel E, Pannekoek H, Horrevoets AJ. 2002. Prolonged fluid shear stress induces a distinct set of endothelial cell genes, most specifically lung Kruppel-like factor (KLF2). Blood 100: 1689-1698.

Dumont DJ, Jussila L, Taipale J, Lymboussaki A, Mustonen T, Pajusola K, Breitman M, Alitalo K. 1998. Cardiovascular failure in mouse embryos deficient in VEGF receptor-3. Science 282: 946-949.

Francois M, Caprini A, Hosking B, Orsenigo F, Wilhelm D, Browne C, Paavonen K, Karnezis T, Shayan R, Downes M, et al. 2008. Sox18 induces development of the lymphatic vasculature in mice. Nature 456: 643-647.

Ginsburg D, Handin RI, Bonthron DT, Donlon TA, Bruns GA, Latt SA, Orkin SH. 1985. Human von Willebrand factor (vWF): isolation of complementary DNA (cDNA) clones and chromosomal localization. Science 228: 1401-1406.

Gittenberger-De Groot AC, Van Den Akker NM, Bartelings MM, Webb S, Van Vugt JM, Haak MC. 2004. Abnormal lymphatic development in trisomy 16 mouse embryos precedes nuchal edema. Dev Dyn 230: 378-384.

Harvey NL, Srinivasan RS, Dillard ME, Johnson NC, Witte MH, Boyd K, Sleeman MW, Oliver G. 2005. Lymphatic vascular defects promoted by Proxl haploinsufficiency cause adultonset obesity. Nat Genet 37: 1072-1081.

Hellstrom M, Phng LK, Hofmann JJ, Wallgard E, Coultas L, Lindblom P, Alva J, Nilsson AK, Karlsson L, Gaiano N, et al. 2007. Dll4 signalling through Notch1 regulates formation of tip cells during angiogenesis. Nature 445: 776-780.

Kampmeier OF. 1928. The genetic history of the valves in the lymphatic system of man. Am J Anat 40: 413-457.

Kampmeier OF. 1969. Evolution and comparative morphology of the lymphatic system. Charles C. Thomas, Springfield, IL.

Kisanuki YY, Hammer RE, Miyazaki J, Williams SC, Richardson JA, Yanagisawa M. 2001. Tie2-Cre transgenic mice: a new model for endothelial cell-lineage analysis in vivo. Dev Biol 230: 230-242.

Lavado A, Lagutin OV, Chow LM, Baker SJ, Oliver G. 2010. Proxl is required for granule cell maturation and intermediate progenitor maintenance during brain neurogenesis. PLOS Biol 8: e1000460. doi: 10.1371/journal.pbio.1000460.

Lee JS, Yu Q, Shin JT, Sebzda E, Bertozzi C, Chen M, Mericko P, Stadtfeld M, Zhou D, Cheng L, et al. 2006. Klf2 is an essential regulator of vascular hemodynamic forces in vivo. Dev Cell 11: 845-857.

Lewis. 1905. The devlopment of the lymphatic system in rabbits. Am J Anat 5: 95-111.
Muzumdar MD, Tasic B, Miyamichi K, Li L, Luo L. 2007. A global double-fluorescent Cre reporter mouse. Genesis 45: 593-605.

Norrmen C, Ivanov KI, Cheng J, Zangger N, Delorenzi M, Jaquet M, Miura N, Puolakkainen P, Horsley V, Hu J, et al. 2009. FOXC2 controls formation and maturation of lymphatic collecting vessels through cooperation with NFATc1. J Cell Biol 185: 439-457.

Oliver G, Harvey N. 2002. A stepwise model of the development of lymphatic vasculature. Ann N Y Acad Sci 979: 159-165.

Oliver G, Srinivasan RS. 2008. Lymphatic vasculature development: current concepts. Ann N Y Acad Sci 1131: 75-81.

Petrova TV, Karpanen T, Norrmen C, Mellor R, Tamakoshi T, Finegold D, Ferrell R, Kerjaschki D, Mortimer P, Yla-Herttuala S, et al. 2004. Defective valves and abnormal mural cell recruitment underlie lymphatic vascular failure in lymphedema distichiasis. Nat Med 10: 974-981.

Risebro CA, Searles RG, Melville AA, Ehler E, Jina N, Shah S, Pallas J, Hubank M, Dillard M, Harvey NL, et al. 2009. Prox1 maintains muscle structure and growth in the developing heart. Development 136: 495-505.

Sabin FR. 1902. On the development of the lymphatic system from the veins and the development of the lymph hearts and thoracic duct in the pig. Am I Anat 1: 367-389.

Soriano P. 1999. Generalized lacZ expression with the ROSA26 Cre reporter strain. Nat Genet 21: 70-71.

Srinivas S, Watanabe T, Lin CS, William CM, Tanabe Y, Jessell TM, Costantini F. 2001. Cre reporter strains produced by targeted insertion of EYFP and ECFP into the ROSA26 locus. BMC Dev Biol 1: 4. doi: 10.1186/1471-213X-1-4.

Srinivasan RS, Dillard ME, Lagutin OV, Lin FJ, Tsai S, Tsai MJ, Samokhvalov IM, Oliver G. 2007. Lineage tracing demonstrates the venous origin of the mammalian lymphatic vasculature. Genes Dev 21: 2422-2432.

Srinivasan RS, Geng X, Yang Y, Wang Y, Mukatira S, Studer M, Porto MP, Lagutin O, Oliver G. 2010. The nuclear hormone receptor Coup-TFII is required for the initiation and early maintenance of Prox 1 expression in lymphatic endothelial cells. Genes Dev 24: 696-707.

Takamoto N, You LR, Moses K, Chiang C, Zimmer WE, Schwartz RJ, DeMayo FJ, Tsai MJ, Tsai SY. 2005. COUP-TFII is essential for radial and anteroposterior patterning of the stomach. Development 132: 2179-2189.

Uhrin P, Zaujec J, Breuss JM, Olcaydu D, Chrenek P, Stockinger H, Fuertbauer E, Moser M, Haiko P, Fassler R, et al. 2010. Novel function for blood platelets and podoplanin in developmental separation of blood and lymphatic circulation. Blood 115: 3997-4005.

van der Putte SC. 1975. The early development of the lymphatic system in mouse embryos. Acta Morphol Neerl Scand 13: 245-286.

Wigle JT, Chowdhury K, Gruss P, Oliver G. 1999. Prox1 function is crucial for mouse lens-fibre elongation. Nat Genet 21: 318-322. 


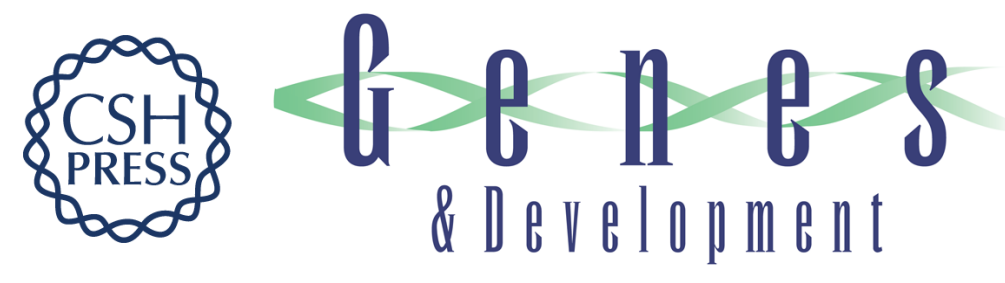

\section{Prox1 dosage controls the number of lymphatic endothelial cell progenitors and the formation of the lymphovenous valves}

R. Sathish Srinivasan and Guillermo Oliver

Genes Dev. 2011, 25:

Access the most recent version at doi:10.1101/gad.16974811

Supplemental
Material http://genesdev.cshlp.org/content/suppl/2011/10/19/25.20.2187.DC1

References This article cites 30 articles, 11 of which can be accessed free at:

http://genesdev.cshlp.org/content/25/20/2187.full.html\#ref-list-1

License

Email Alerting

Receive free email alerts when new articles cite this article - sign up in the box at the top

Service

right corner of the article or click here.

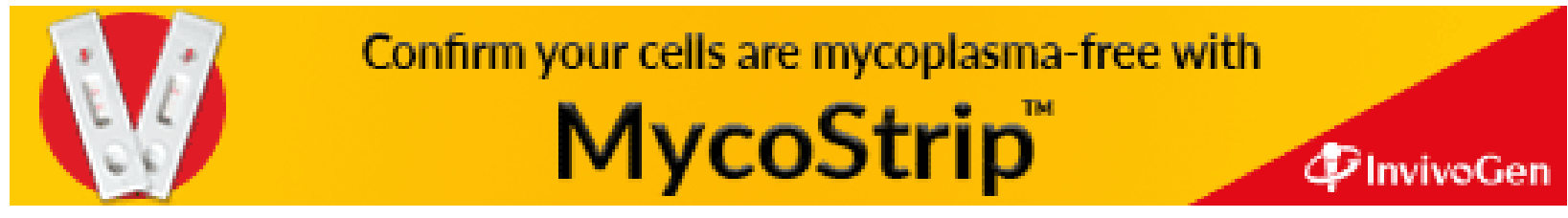

